\title{
An Optimal Resource Reuse Strategy for QoS-Awared Device-to-Device Communications
}

\author{
Daohua Zhu ${ }^{1,2}$, Jiaheng Wang ${ }^{1}$, A. Lee Swindlehurst ${ }^{2}$, Chunming Zhao ${ }^{1}$ \\ ${ }^{1}$ National Mobile Communications Research Laboratory, \\ Southeast University, Nanjing 210096, China \\ ${ }^{2}$ Center for Pervasive Communications and Computing, \\ University of California, Irvine, CA 92697-2625
}

\begin{abstract}
Device-to-device (D2D) communication is a promising technique for improving the spectral efficiency of cellular networks. In this paper, we consider joint optimization of pair assignment and power control for D2D pairs reusing downlink cellular resources, and aim to maximize the aggregate rate of D2D communications while guaranteeing the quality of service (QoS) of the cellular users. By transforming the original difficult problem into an equivalent form, we are able to analytically characterize the optimal power control and pair assignment for the D2D and cellular users. Based on these results, we then propose an efficient algorithm to obtain the jointly optimal power control and pair assignment strategy. The numerical results show the superiority of our proposed method over existing ones.
\end{abstract}

Keywords-Device-to-Device communication, spectral efficiency, power control, cellular communication, quality of service, joint pair assignment and power control.

\section{INTRODUCTION}

D2D communication, which allows two users in close proximity to discover and communicate with each other, arises as an important supplement to cellular networks. Compared with relaying through a third party, D2D communication possesses several advantages, including short latency, power efficiency, more physical layer security [1], etc. Therefore, D2D techniques have received much attention and been proposed to be incorporated into current and future wireless networks [2].

Flexible and efficient resource sharing strategies are an intensive research topic for underlay D2D communication [3][6]. Particularly, the sum rate maximization of one D2D link and one cellular user (CU) has been studied in [3], while [4] proposed heuristic resource reuse methods to address the interference mitigation. Meanwhile, [5] proposed an interference limited area scheme to select proper CUs for D2D pair reuse. A graph-based method was considered in [6] to allocate resources with D2D pairs.

In this study, we consider multiple D2D pairs who reuse the downlink resources of multiple CUs in a cellular network. Reusing downlink resources can prevent the near-far effect that often arises in the uplink reuse scheme, where D2D pairs close to the base station (BS) may cause strong interference to CUs. We allow each D2D pair to reuse multiple CUs' resources and therefore our design is more general than [4], [6]. Since D2D communication is a complement to cellular communication, CUs generally have higher priorities and their QoS must be properly protected [2], [3]. To this end, we formulate a throughput maximization problem for the D2D pairs with QoS requirements for the CUs. Our goal is to jointly optimize the assignment of the D2D pairs to each resource and the power control of both D2D pairs and CUs so as to maximize the throughput of all D2D pairs while providing a minimum QoS for each CU.

Due to the nonconvex and combinatorial nature of the formulated problem, finding the optimal strategy is challenging. By revealing the power coordination of a hybrid network, we obtain an equivalent transformation of formulated problem, which is convex with proper relaxation. Based on problem transformation, we characterize the optimal power control and pair assignment given that the other is fixed. Then, we propose a joint power control and pair assignment algorithm to solve the relaxed problem, which is statistically optimal for the original one. The numerical results verify the statistical optimality and show our proposed resource reuse strategy holds a superiority over existing D2D schemes.

\section{System Model AND PROBLEM Formulation}

In this section, we consider a hybrid single-cell network as shown in Fig. 1, which consists of $N$ orthogonal CUs and $D$ D2D pairs. All CUs are in the downlink phase while D2D pairs can reuse their cellular resources to establish local communications. The base station (BS), as a centralized controller, takes charge of assigning a D2D pair to reuse the cellular resources and coordinates the transmit power of both CUs and D2D pairs. Let $x_{d, n}=1$ indicate when the D2D pair $d$ reuses the resource of CU $n$, otherwise $x_{d, n}=0$. Each CU's resource can be reused by at most one D2D pair, i.e.,

$$
x_{d, n} \in\{0,1\}, \forall d, n ; \quad \sum_{d=1}^{D} x_{d, n} \leq 1, \forall n .
$$

This constraint is convenient for management and billing of D2D communications and has been adopted in [4] and [6].

Without loss of generality, we introduce the normalized (by the noise power) gains $g_{n}, g_{d, n}, g_{n, d}^{I}$, and $g_{d, n}^{I}$ to represent the normalized channel gains of the following channels: the transmission channel of CU $n$ on the $n$th resource, the transmission channel of D2D pair $d$ on the $n$th resource, the cross channel from BS to D2D pair $d$, and the cross channel from the D2D transmitter to $\mathrm{CU} n$. Hence, the data rate of $\mathrm{D} 2 \mathrm{D}$ pair $d$ is given by

$$
r_{d}=\sum_{n=1}^{N} x_{d, n} \log _{2}\left(1+\frac{p_{d, n} g_{d, n}}{1+g_{n, d}^{I} p_{n}}\right),
$$




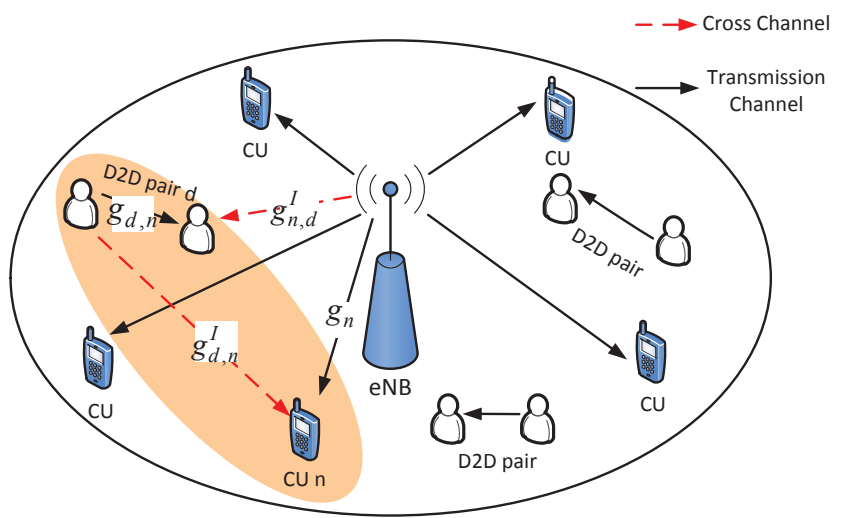

Fig. 1. D2D communications underlaying downlink cellular network

where $p_{d, n} \geq 0$ and $p_{n} \geq 0$ are the transmit power of D2D pair $d$ and $\mathrm{CU} n$ on the associated resource.

D2D communication is typically implemented as a complement to cellular communication, so CUs generally have higher priorities than D2D pairs. Thus, we impose a QoS target for each CU to protect the traditional cellular communication:

$$
\sum_{d=1}^{D} x_{d, n} \log _{2}\left(1+\frac{p_{n} g_{n}}{1+g_{d, n}^{I} p_{d, n}}\right) \geq R_{n}, \forall n .
$$

Our goal is to improve the overall throughput of D2D pairs while satisfying the cellular QoS constraints by properly allocating the D2D pairs and the D2D transmission power among the $N$ resources. Such a design problem can be formulated as

$$
\begin{aligned}
\underset{\left\{x_{d, n}, p_{d, n}, p_{n}\right\}}{\max .} & \sum_{d=1}^{D} r_{d} \\
\text { s.t. } & \sum_{d=1}^{D} x_{d, n} \log _{2}\left(1+\frac{p_{n} g_{n}}{1+g_{d, n}^{I} p_{d, n}}\right) \geq R_{n}, \forall n, \\
& x_{d, n} \in\{0,1\} \forall d, n ; \sum_{d=1}^{D} x_{d, n} \leq 1, \forall n \\
& \sum_{n=1}^{N} x_{d, n} p_{d, n} \leq P_{d}, \forall d \\
& \sum_{n=1}^{N} p_{n} \leq P_{B S} .
\end{aligned}
$$

The BS is to maintain reliable connections with the CUs under the power budget $P_{B S}$. Meanwhile, each D2D pair $d$ reuses the cellular resources under the power budget $P_{d}$.

\section{Optimal Pair Assignment And Power Control SCHEME}

The formulated problem (4) is a combinatorial optimization problem due to the discrete variables $\left\{x_{d, n}\right\}$. Besides, neither the objective function (4a) nor the QoS constraints (4b) is jointly concave in $\left\{p_{d, n}, p_{n}\right\}$, making it even harder to solve. Nevertheless, we find that (4a) can be transformed into an equivalent optimization problem that possesses favorable properties for finding the optimal pair assignment and power control.

The equivalent transformation is provided in the following Proposition 1. Moreover, we also prove in Lemma 1 which shows that the equivalent problem becomes a convex problem if the time-sharing relaxation is applied.

Proposition 1. Let $\alpha_{d, n}=g_{n} g_{d, n}, \beta_{d, n}=g_{n}+\omega_{n} g_{n, d}^{I}$ and $\gamma_{d, n}=\omega_{n} g_{n, d}^{I} g_{d, n}^{I}$. Then, (4) is equivalent to the following problem

$$
\begin{aligned}
\underset{\left\{p_{d, n}, x_{d, n}\right\}}{\max } & \sum_{d=1}^{D} \sum_{n=1}^{N} x_{d, n} \log _{2}\left(1+\frac{\alpha_{d, n} p_{d, n}}{\beta_{d, n}+\gamma_{d, n} p_{d, n}}\right) \\
\text { s.t. } & x_{d, n} \in\{0,1\}, \forall d, n, \sum_{d=1}^{D} x_{d, n} \leq 1, \forall n, \\
& \sum_{n=1}^{N} x_{d, n} p_{d, n} \leq P_{d}, \forall d, \\
& \sum_{n=1}^{N} \sum_{d=1}^{D} \frac{\omega_{n}}{g_{n}} g_{d, n}^{I} x_{d, n} p_{d, n} \leq P_{B S}-\sum_{n=1}^{N} \frac{\omega_{n}}{g_{n}},
\end{aligned}
$$

where $\omega_{n} \triangleq 2^{R_{n}}-1$.

Proof: Supposing the D2D pair $d$ reuses the CU $n$ 's resource (i.e., $x_{d, n}=1$ ), then from QoS constraint (4b), we have $p_{n} \geq \omega_{n}\left(1+g_{d, n}^{I} p_{d, n}\right) / g_{n}$. Observe that for fixed $p_{d, n}$, the objective function defined in (4a) is monotonically decreasing in $p_{n}$. Therefore the optimal $p_{n}$ must be achieved at $p_{n}^{\star}=\omega_{n}\left(1+g_{d, n}^{I} p_{d, n}\right) / g_{n}$. Then, we can substitute $p_{n}^{\star}$ into the objective function and obtain its equivalent form (5a). Similarly, power constraint (5b) is obtained by substituting $p_{n}^{\star}$ into (4e), which implies that the interference power should not surpass the remaining power of the BS.

The equivalent form in (5) reduces the original variables from $\left\{p_{d, n}, x_{d, n}, p_{n}\right\}$ to $\left\{p_{d, n}, x_{d, n}\right\}$ and removes QoS constraint (4b). Although (5) is still combinatorial, we show in Lemma 1 that, unlike (4), the relaxed problem is convex.

Lemma 1. Relax $x_{d, n}$ to the interval $[0,1]$ and introduce $s_{d, n}=x_{d, n} p_{d, n}$. Then the relaxed form of problem (5) becomes

$$
\begin{aligned}
\underset{\left\{s_{d, n}, x_{d, n}\right\}}{\max } & \sum_{d=1}^{D} \sum_{n=1}^{N} x_{d, n} \log _{2}\left(1+\frac{\alpha_{d, n} \frac{s_{d, n}}{x_{d, n}}}{\beta_{d, n}+\gamma_{d, n} \frac{s_{d, n}}{x_{d, n}}}\right) \\
\text { s.t. } & x_{d, n} \in[0,1], \forall d, n, \sum_{d=1}^{D} x_{d, n} \leq 1, \forall n, \\
& \sum_{n=1}^{N} s_{d, n} \leq P_{d}, \forall d, \\
& \sum_{n=1}^{N} \sum_{d=1}^{D} \frac{\omega_{n}}{g_{n}} g_{d, n}^{I} s_{d, n} \leq P_{B S}-\sum_{n=1}^{N} \frac{\omega_{n}}{g_{n}},
\end{aligned}
$$

which is a convex optimization problem.

Proof: Actually, such relaxation is so-called time-sharing relaxation [7], which in practical indicates multiple D2D pairs 
may share the same resource in a TDMA (time division multiple access) manner. It is easy to see that all the constraints of the relaxed problem are jointly convex in $\left\{s_{d, n}, x_{d, n}\right\}$. It remains to verify the concavity of objective function (6a). Fortunately, (6a) can be seen as a perspective function of a concave function, therefore it is concave. To prove this, we first rewrite one term of the relaxed objective function below

$$
\begin{aligned}
g\left(x_{d, n}, s_{d, n}\right) & =x_{d, n} f\left(\frac{s_{d, n}}{x_{d, n}}\right) \\
& =x_{d, n} \log _{2}\left(1+\frac{\alpha_{d, n} \frac{s_{d, n}}{x_{d, n}}}{\beta_{d, n}+\gamma_{d, n} \frac{s_{d, n}}{x_{d, n}}}\right) .
\end{aligned}
$$

The function $g\left(x_{d, n}, s_{d, n}\right)$ is the perspective function

$$
f\left(s_{d, n}\right)=\log _{2}\left(1+\frac{\alpha_{d, n} s_{d, n}}{\beta_{d, n}+\gamma_{d, n} s_{d, n}}\right) .
$$

In addition, let $h\left(s_{d, n}\right)=\alpha_{d, n} s_{d, n} /\left(\beta_{d, n}+\gamma_{d, n} s_{d, n}\right)$, whose second derivative is

$$
h^{\prime \prime}\left(s_{d, n}\right)=-\frac{2 \alpha_{d, n} \beta_{d, n} \gamma_{d, n}}{\left(\beta_{d, n}+\gamma_{d, n} s_{d, n}\right)^{3}} \leqslant 0,
$$

indicating that $h\left(s_{d, n}\right)$ is a concave function. Given that $\log (1+h)$ is nondecreasing in $h$ and concave, $f\left(s_{d, n}\right)$ is a concave function and therefore $g\left(x_{d, n}, s_{d, n}\right)$ is concave [8]. Finally, the objective function (6a) is concave, since it is a nonnegative weighted sum of concave functions. result.

Combining Proposition 1 and Lemma 1, we have following

Corollary 1. For fixed $\left\{x_{d, n}\right\},(5)$ is a convex problem whose solution provides the optimal power control.

\section{A. Properties of Optimal Power Control and Pair Assignment}

We have shown that (5) is convex in terms of power control, and thus the power allocation can be optimally and efficiently found using standard methods. An exhaustive search could be performed to allocate the D2D pairs to the resources, but the complexity of the exhaustive search exponentially increases with the number of CUs and D2D pairs, making it computationally prohibitive in practice.

Although we have proved the concavity of the relaxed problem (6), it does not directly lead to pair-resource assignment and power control design. Therefore, we would like to investigate the properties of the optimal pair assignment and power control. To this end, we follow the standard derivation of the KKT conditions of the relaxed problem, which are sufficient and necessary conditions for an optimal solution. Specifically, the Lagrangian of the relaxed problem is

$$
\begin{aligned}
\mathcal{L} & \left(\left\{x_{d, n}\right\},\left\{s_{d, n}\right\}, \boldsymbol{\phi}, \boldsymbol{\mu}, \lambda\right) \\
= & \sum_{d=1}^{D} \sum_{n=1}^{N} x_{d, n} \log _{2}\left(1+\frac{\alpha_{d, n} \frac{s_{d, n}}{x_{d, n}}}{\beta_{d, n}+\gamma_{d, n} \frac{s_{d, n}}{x_{d, n}}}\right)+ \\
& \sum_{n=1}^{N} \phi_{n}\left(1-\sum_{d=1}^{D} x_{d, n}\right)+\sum_{d=1}^{D} \mu_{d}\left(P_{d}-\sum_{n=1}^{N} s_{d, n}\right)+ \\
& \lambda\left(P_{B S}-\sum_{n=1}^{N} \frac{\omega_{n}}{g_{n}}-\sum_{n=1}^{N} \sum_{d=1}^{D} \frac{\omega_{n}}{g_{n}} g_{d, n}^{I} s_{d, n}\right)
\end{aligned}
$$

where $\phi \succeq \mathbf{0}, \boldsymbol{\mu} \succeq \mathbf{0}$ and $\lambda \succeq \mathbf{0}$ are Lagrange multipliers associated with the constraints (6b), (6c) and (6d), respectively. Taking the first derivatives of the Lagrangian with respect to $s_{d, n}$, the optimal power control for given pair assignment shall satisfy

$$
\begin{aligned}
\frac{\partial \mathcal{L}}{\partial s_{d, n}^{\star}} & =\frac{\alpha_{d, n} \beta_{d, n}}{\ln 2\left(\beta_{d, n}+\gamma_{d, n} \frac{s_{d, n}^{\star}}{x_{d, n}}\right)\left[\beta_{d, n}+\left(\alpha_{d, n}+\gamma_{d, n}\right) \frac{s_{d, n}^{\star}}{x_{d, n}}\right]} \\
& -\left(\mu_{d}+\lambda \frac{\omega_{n}}{g_{n}} g_{d, n}^{I}\right)\left\{\begin{array}{l}
=0,0<\frac{s_{d, n}^{\star}}{x_{d, n}} \\
<0,0=\frac{s_{d, n}^{\star}}{x_{d, n}}
\end{array}\right.
\end{aligned}
$$

These conditions can be utilized to find the optimal power control for any given pair assignment, as follows:

$$
p_{d, n}^{\star}=\left(\sqrt{\left(\frac{a_{d, n}^{(1)}}{2 a_{d, n}^{(0)}}\right)^{2}-\frac{a_{d, n}^{(2)}\left(\mu_{d}, \lambda\right)}{a_{d, n}^{(0)}}}-\frac{a_{d, n}^{(1)}}{2 a_{d, n}^{(0)}}\right)^{+}
$$

which is a real positive root of the quadratic equation

$$
a_{d, n}^{(0)} x^{2}+a_{d, n}^{(1)} x+a_{d, n}^{(2)}\left(\mu_{d}, \lambda\right)=0
$$

where $a_{d, n}^{(0)}=\left(\alpha_{d, n}+\gamma_{d, n}\right) \gamma_{d, n}, a_{d, n}^{(1)}=\left(\alpha_{d, n}+2 \gamma_{d, n}\right) \beta_{d, n}$ and $a_{d, n}^{(2)}\left(\mu_{d}, \lambda\right)=\beta_{d, n}^{2}-\beta_{d, n} \alpha_{d, n} /\left(\ln 2\left(\mu_{d}+\lambda \omega_{n} / g_{n} g_{d, n}^{I}\right)\right)$.

Similarly, the optimal pair-resource assignment for a given power allocation can also be derived by taking the first-order derivatives of $\mathcal{L}$ with respect to $x_{d, n}$ as follows

$$
\begin{aligned}
\frac{\partial \mathcal{L}}{\partial x_{d, n}^{\star}} & =\log _{2}\left(1+\frac{\alpha_{d, n} \frac{s_{d, n}}{x_{d, n}^{\star}}}{\beta_{d, n}+\gamma_{d, n} \frac{s_{d, n}}{x_{d, n}^{\star}}}\right) \\
& -\frac{\alpha_{d, n} \beta_{d, n} \frac{s_{d, n}}{x_{d, n}^{\star}}}{\ln 2\left(\beta_{d, n}+\gamma_{d, n} \frac{s_{d, n}}{x_{d, n}^{\star}}\right)\left[\beta_{d, n}+\left(\alpha_{d, n}+\gamma_{d, n}\right) \frac{s_{d, n}}{x_{d, n}^{\star}}\right]} \\
& -\phi_{n}\left\{\begin{array}{l}
<0, x_{d, n}^{\star}=0 \\
=0,0<x_{d, n}^{\star}<1, \\
>0, x_{d, n}^{\star}=1
\end{array}\right.
\end{aligned}
$$

for $d=1,2, \ldots, D$ and $n=1,2, \ldots, N$. Let

$$
\begin{aligned}
& U_{d, n}\left(p_{d, n}^{\star}\right)=\log _{2}\left(1+\frac{\alpha_{d, n} p_{d, n}^{\star}}{\beta_{d, n}+\gamma_{d, n} p_{d, n}^{\star}}\right)- \\
& \frac{\alpha_{d, n} \beta_{d, n} p_{d, n}^{\star}}{\ln 2\left(\beta_{d, n}+\gamma_{d, n} p_{d, n}^{\star}\right)\left[\beta_{d, n}+\left(\alpha_{d, n}+\gamma_{d, n}\right) p_{d, n}^{\star}\right]},
\end{aligned}
$$

which can be interpreted as the utility or benefit of D2D pair $d$ reusing CU $n$ 's resource. Therefore, only the $\mathrm{D} 2 \mathrm{D}$ pair with the largest $U_{d, n}$ can reuse $\mathrm{CU} n$ 's resource, i.e.,

$$
x_{\tilde{d}, n}^{\star}=1, \tilde{d}=\underset{1 \leq d \leq D}{\operatorname{argmax}} U_{d, n} ; x_{d, n}^{\star}=0, \forall d \neq \tilde{d},
$$

or multiple $\mathrm{D} 2 \mathrm{D}$ pairs with the same maximal $U_{d, n}$ share this resource in a Time Division Multiple Access (TDMA) manner. Note that the time-sharing proportions of the D2D pairs should satisfy (6b). From (15), $U_{d, n}$ mainly depends on different channel gains, which are independent continuousvalued random variables due to the various locations of the CUs and D2D pairs. Consequently, the possibility of the event 
$U_{d_{1}, n}=U_{d_{2}, n}\left(d_{1} \neq d_{2}\right)$ is zero in practice. Therefore, although $x_{d, n}$ is relaxed to $[0,1]$, the obtained pair assignments are still integer indications. This property is also verified by the numerical results. To conclude, we have following result.

Corollary 2. The optimal solution of (5) can be obtained by solving the relaxed problem (6) with probability one.

Even if there exist non-integer $x_{d, n} \mathrm{~s}$ ', we can round them and solve the convex power control problem in Corollary 1.

\section{B. Joint Optimization of Power Control and Pair Assignment}

Using (12) and (15), we can determine the power and associated pair assignment according to (12) and (14), which are continuous with dual variables $\{\lambda, \boldsymbol{\mu}\}$. As a consequence, it is more convenient to solve the relaxed problem than (5). In following, we propose an algorithm to find the optimal $\{\lambda, \boldsymbol{\mu}\}$ to obtain the joint optimal pair assignment and power control. It is worth mentioning that (5) and (6) are feasible only if the RHS (Right Hand Side) of constraint (5d) is positive, i.e., the BS must have surplus power to overcome the interference from the D2D pairs.

Our proposed algorithm consists of two nested loops: the outer loop (Algorithm 1) and the inner loop (Algorithm 2) for updating $\lambda$ and $\boldsymbol{\mu}$ associated with the power constraint of the BS and D2D pairs, respectively. From (12), we know that the optimal power $p_{d, n}^{\star}$ is decreasing in $\lambda$, so the bisection method can efficiently find the optimal $\lambda^{\star}$.

In Algorithm 1, we first carry out the pair assignment and power control to find the optimal $\boldsymbol{\mu}^{\star}$ for given $\lambda$. Then, we calculate a power increment to overcome interference $P^{r e}=\sum_{n=1}^{N} \sum_{d=1}^{D} \frac{\omega_{n}}{g_{n}} g_{d, n}^{I} x_{d, n} p_{d, n}$ and compare it with the remaining power of the BS: $P^{s u r}=P_{B S}-\sum_{n=1}^{N} \omega_{n} / g_{n}$ to decide whether to increase $\lambda$ or not.

In Algorithm 2, we demonstrate how to determine the set of optimal Lagrangian multipliers $\left\{\mu_{1}^{\star}, \mu_{2}^{\star}, \ldots, \mu_{D}^{\star}\right\}$ for the given $\lambda$. The basic idea is to adjust $\left\{\mu_{1}, \mu_{2}, \ldots, \mu_{D}\right\}$ in order to fulfill the power constraints of the D2D pairs. This follows from the complementary slackness condition

$$
\sum_{d=1}^{D} \xi_{d}^{\star}\left(P_{d}-\sum_{n=1}^{N} x_{d, n}^{\star} p_{d, n}^{\star}\right)=0 .
$$

That is, if $\xi_{d}^{\star}>0$, we have $\left(P_{d}-\sum_{n=1}^{N} x_{d, n}^{\star} p_{d, n}^{\star}\right)=0$, which can be used to find the optimal $\xi_{d}^{\star}$ via the bisection method; if $\left(P_{d}-\sum_{n=1}^{N} x_{d, n}^{\star} p_{d, n}^{\star}\right)>0$, then $\xi_{d}^{\star}$ must be zero.

Algorithm 2 initializes the search for $\boldsymbol{\mu}$ from 0 . If the initially obtained $\left\{p_{d, n}, x_{d, n}\right\}$ can guarantee satisfaction of (17) for all the D2D pairs, the algorithm is terminated. Otherwise, it will gradually increase $\mu_{d}$ for the D2D pair with the largest deviation from the power constraint until the conditions (17) for all D2D pairs are satisfied. From the conditions in (14), we find that the larger $U_{d, n}$ is, the more likely D2D pair $d$ would reuse CU $n$ 's resource. Moreover, since $p_{d, n}^{\star}$ is monotonically decreasing with respect to $\mu_{d}$ and $U_{d, n}$ is an increasing function of $p_{d, n}^{\star}, U_{d, n}$ is decreasing in $\mu_{d}$. Hence, increasing $\mu_{d}$ would lower $p_{d, n}^{\star}$ and lead to a smaller $U_{d, n}$, thus forcing D2D pair $d$ not to reuse resource $n$.

$\overline{\text { Algorithm } 1 \text { Joint Optimization of Pair Assignment and Power }}$
Control

Input: $P_{B S} ; P_{d}, \forall d ; R_{n}, g_{n} \forall n ; g_{d, n}, g_{d, n}^{I}, g_{n, d}^{I}, \forall n, d$.

Output: $x_{d, n}^{\star} p_{d, n}^{\star} \forall d, n$.

Initialization: $\epsilon=$ a small positive value; $l_{b}=0, u_{b}=$ $\lambda_{\max } ; k=1, \lambda_{k}=\left(l_{b}+u_{b}\right) / 2$. Let $P^{\text {sur }}=P_{B S}-$ $\sum_{n=1}^{N} \omega_{n} / g_{n}$

While $\left|u_{b}-l_{b}\right|>\epsilon$

1. Obtain optimal $\boldsymbol{\mu}^{\star}$ and $\left\{p_{d, n}^{\star}, x_{d, n}^{\star}\right\}$ for given $\lambda_{k}$ using Algorithm 2.

2. Compute $P^{r e}=\sum_{n=1}^{N} \sum_{d=1}^{D} \frac{\omega_{n}}{g_{n}} g_{d, n}^{I} x_{d, n} p_{d, n}$.

3. If $P^{r e}<P_{B S}^{\text {sur }}, u_{b}=\lambda_{k}$, otherwise, $l_{b}=\lambda_{k}$.

4. Update $\lambda_{k+1}=\left(l_{b}+u_{b}\right) / 2, k=k+1$.

EndWhile

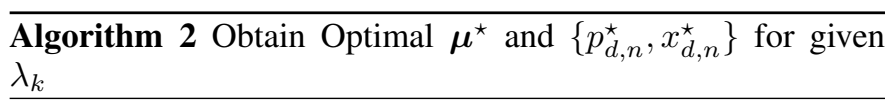

Initialize $\boldsymbol{\mu}=\mathbf{0}$. Compute $p_{d, n}, \forall d, n$ with $\lambda_{k}, \boldsymbol{\mu}$ via (12). Substitute $p_{d, n}$ into $U_{d, n}$ and assign D2D pairs to each CU according to (16).

While $\sum_{n=1}^{N} x_{d, n} p_{d, n}>P_{d}$ for any $d$ :

1: $P_{d}^{\prime}=\sum_{n=1}^{N} x_{d, n} p_{d, n}-P_{d}, \tilde{d}=\operatorname{argmax}_{1 \leq d \leq D} P_{d}^{\prime}$.

2: Let $\mathcal{N}_{\tilde{d}}=\left\{n \mid x_{\tilde{d}, n}>0\right\}$ be the set of resources that D2D pair $d$ can reuse.

3: While $\sum_{n=1}^{N} x_{\tilde{d}, n} p_{\tilde{d}, n}>P_{\tilde{d}}$

4: $\quad$ For all $n \in \mathcal{N}_{\tilde{d}}$

5: $\quad d(n)=\operatorname{argmax}_{1<d<D, d \neq \tilde{d}} U_{d, n}$.

6: $\quad$ Compute $\mu_{\tilde{d}}^{(n)}$ such that $U_{\tilde{d}, n}=U_{d(n), n}(1-\epsilon)$.

7: EndFor

8: Let $m=\operatorname{argmin}_{n \in \mathcal{N}_{\tilde{d}}} \mu_{\tilde{d}}^{(n)}, \mu_{\tilde{d}}=\mu_{\tilde{d}}^{(m)}, x_{\tilde{d}, m}=0$, and $x_{d(m), m}=1$. Update $P_{\tilde{d}}^{\prime}=\sum_{n=1}^{N} x_{\tilde{d}, n} p_{\tilde{d}, n}$.

9: $\quad$ If $P_{\tilde{d}}^{\prime}<P_{\tilde{d}}$

10: $\quad \underline{P}_{\tilde{d}}^{\prime}=P_{\tilde{d}}^{\prime}+p_{\tilde{d}, m}$.

11: If $\underline{P}_{\tilde{d}}^{\prime}>P_{\tilde{d}}$

12: $\quad x_{\tilde{d}, m}=\left(P_{\tilde{d}}-P_{\tilde{d}}^{\prime}\right) / p_{\tilde{d}, m}, x_{d(m), m}=\left(1-x_{\tilde{d}, m}\right)$.

13: $\quad$ ElseIf $\underline{P}_{\tilde{d}}^{\prime}<P_{\tilde{d}}$

14: $\quad x_{\tilde{d}, m}=1, x_{d(m), m}=0$.

14: $\quad$ Compute $\mu_{\tilde{d}}$ such that $\sum_{n=1}^{N} x_{\tilde{d}, n} p_{\tilde{d}, n}\left(\mu_{\tilde{d}}, \lambda\right)=P_{\tilde{d}}$. 15: EndIf

16: EndIf

17: EndWhile

EndWhile

To appropriately choose the search range of $(\lambda, \boldsymbol{\mu})$ in Algorithms 1 and 2, we have following result in Lemma 2.

Lemma 2. Assume problem (5) is feasible and denote the optimal Lagrange multipliers by $\left(\lambda^{\star}, \boldsymbol{\mu}^{\star}\right)$. Then

$$
\begin{aligned}
& 0<\lambda^{\star}<\lambda^{\max }=\max _{1 \leq d \leq D, 1 \leq n \leq N} \frac{\alpha_{d, n} g_{n}}{\ln 2 \beta_{d, n} g_{d, n}^{I} \omega_{n}} . \\
& 0<\mu_{d}^{\star}<\mu_{d}^{\max }=\max _{1 \leq n \leq N} \frac{\alpha_{d, n}}{\ln 2 \beta_{d, n}}, \forall d .
\end{aligned}
$$

Proof: From (12), there is at least one $p_{d, n}^{\star} \geq 0$ if and only if $\beta_{d, n}-\frac{\alpha_{d, n}}{\ln 2\left(\lambda \frac{\omega_{n}}{g_{n}}+\mu_{d}\right)}<0, \forall d, n$. Letting $\mu_{d}=0$ or $\lambda=0$, 


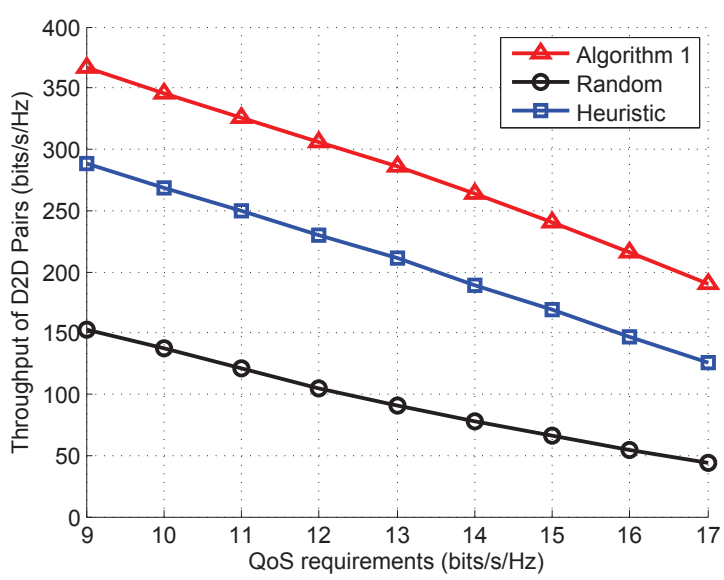

Fig. 2. Throughput of the D2D pairs versus QoS requirements with 20 D2D pairs and 30 CUs.

we have $\lambda^{\max }$ and $\mu_{d}^{\max }$, respectively. Based on the feasibility assumption, at least one $p_{d, n}$ is positive or equals zero.

\section{NumericAl RESUlts}

In this section, we present numerical results to evaluate the performance of our proposed algorithm. We label the algorithm proposed in [4] and a random reuse scheme as 'Heuristic' and 'Random' for comparison. Our simulation parameters can be found in the Urban Macro (UMa) model [9], with cell radius $500 \mathrm{~m}$ and thermal noise level $-174 \mathrm{dBm} / \mathrm{Hz}$. The power budgets of the BS and D2D pairs are chosen as $46 \mathrm{dBm}$ and $20 \mathrm{dBm}$, respectively. The CUs and D2D pairs are randomly distributed within the cell. The D2D TX and RX are all separated by a distance of $20 \mathrm{~m}$.

From Fig. 2, we find that as the QoS requirement increases (i.e., the leftover power of the BS decreases), the throughput of all D2D pairs deteriorates, since the capability of overcoming the interference is weakened. As can be seen in Fig. 3, when the number of CUs increases, the D2D pairs have more available resources to reuse, thus leading to higher sum rates. One can observe that our proposed algorithm has a significant advantage over the other methods. The number of continuous optimal $\left\{x_{d, n}\right\}$ solutions in both figures is zero, which confirms the conclusion drawn in Corollary 2.

\section{CONCLUSion}

In this paper, we formulated a joint pair-resource assignment and power control problem to maximize the sum rate of D2D communications while guaranteeing the QoS of CUs. We obtained an equivalent transformation of the formulated problem, which is convex with proper relaxation. Based on the relaxed problem, we derived the optimal power control and characterized the optimal pair assignment. Then, we proposed an efficient algorithm for joint optimization of the power control and pair-resource assignment. The numerical results showed our proposed resource reuse strategy provides superior performance compared with existing methods.

\section{ACKNOWLEDGMENT}

This work was supported by the 973 Program (2013CB336600 and 2013CB329203), National Natural

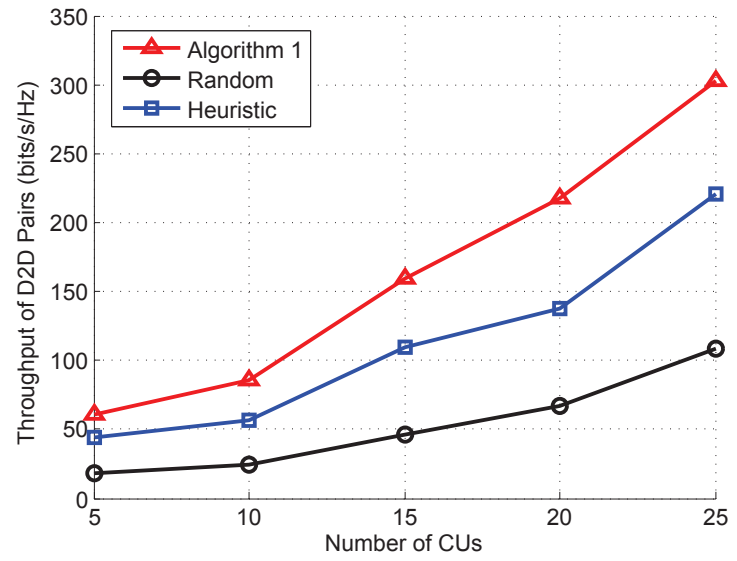

Fig. 3. Throughput of the D2D pairs versus the number of CUs with 20 D2D pairs and QoS requirements at 10bits/s/Hz.

Science Foundation of China (61201174), Natural Science Foundation of Jiangsu (BK2012325), the Fundamental Research Funds for the Central Universities, and the China Scholarship Council.

\section{REFERENCES}

[1] D. Zhu, A. L. Swindlehurst, S. Ali. A. Fakoorian, W. Xu, and C. M. Zhao, "Device-to-device communications: the physical layer security advantage," in Proc. IEEE ICCASP 2014, May 2014, pp. 1-5.

[2] P. Phunchongharn, E. Hossain, D. I. Kim, "Resource allocation for device-to-device communications underlaying LTE-advanced networks," IEEE Wireless Commun., vol. 20, no. 4, pp. 91-100, Aug. 2013.

[3] C.-H. Yu, K. Doppler, C. B. Ribeiro, and O. Tirkkonen, "Resource sharing optimization for device-to-device communication underlaying cellular networks," IEEE Trans. Wireless Commun., vol. 10, no. 8, pp. 2752-2763, Aug. 2011.

[4] M. Zulhasnine, C. Huang, and A. Srinivasan, "Efficient resource allocation for device-to-device communication underlaying LTE network," in Proc. IEEE WiMob 2010, Oct. 2010, pp. 368-375.

[5] H. Min, J. Lee, S. Park, and D. Hong, "Capacity enhancement using an interference limited area for device-to-device uplink underlaying cellular networks," IEEE Trans. Wireless Commun., vol. 10, no. 12, pp. 3995-4000, Dec. 2011.

[6] D. Feng, L. Lu, Y. Yuan-Wu, G. Y. Li, G. Feng, and S. Li, "Deviceto-device communications underlaying cellular networks," IEEE Trans. Commun., vol. 61, no. 8, pp. 3541-3551, Aug. 2013.

[7] C. Y. Wong, R. S. Cheng, K. B. Lataief, and R.D. Murch, "Multiuser OFDM with adaptive subcarrier, bit, and power allocation," IEEE J. Sel. Areas Commun., vol. 17, no. 10, pp. 1747-1758, Oct. 1999.

[8] S. Boyd and L. Vandenberghe, Convex Optimization. Cambridge, U. K.: Cambridge University Press, 2004.

[9] Guidelines for evaluation of radio interface technologies for IMTAdvanced, Rep. M.2135-0, 2008. 